\title{
A note on constant curvature solutions in cylindrically symmetric metric $f(R)$ Gravity
}

\author{
D.Momeni计 \\ Department of Physics,Faculty of science, \\ Islamic Azad University, Karaj Branch.Iran, \\ Karaj, Rajaei shahr, P.O.Box: 31485-313 \\ H. Gholizade‡ \\ Department of Engineering Science, \\ Faculty of Engineering, University of Tehran, \\ Tehran, PO Box 11155-4563, Iran
}

(Dated: today)

\begin{abstract}
In the previous work we introduced a new static cylindrically symmetric vacuum solutions in Weyl coordinates in the context of the metric $f(R)$ theories of gravity [1]. Now we obtain a 2parameter family of exact solutions which contains cosmological constant and a new parameter as $\beta$. This solution corresponds to a constant Ricci scalar. We proved that in $f(R)$ gravity , the constant curvature solution in cylindrically symmetric cases is only one member of the most generalized Tian family in GR. We show that our constant curvature exact solution is applicable to the exterior of a string. Sensibility of stability under initial conditions is discussed.
\end{abstract}

\footnotetext{
* Corresponding author

${ }^{\dagger}$ Electronic address: dmomeni@phymail.ut.ac.ir

†Electronic address: gholizade@ut.ac.ir
} 


\section{INTRODUCTION}

Any phenomenal problem in theoretical physics commitment using of any thinkable method to attack. Acceleration of universe in now era is the most challenge for all branch of physics. If we are interested only on attribute it to a matter field, we envisage with different kind of models which we called them physical models or dark fluids. We present one of them as a simple phase transition in a known metric in [9]. Another approach is encroach to geometrical parts of Einstein field equations and working with a most general action. all these different proposals have been put forward with the hope of obtaining a consistent theoretical background to the recent observation of an expanding universe which is seemingly not accessible through the Standard Model of cosmology. Modified or alternative theories of gravity is the paradigm under which all those theories which differ from the Einsteinian gravity are studied. One of these non-Einsteinian theories, that arose a lot of enthusiasm recently, is the so-called $f(R)$ gravity in which a function, $f(R)$, replaces the EinsteinHilbert (gravitational) Lagrangian R. Later Buchdahl published a paper about some Mathematical solutions with this form of action[2]. These theories could be thought of as a special kind of higher derivative gravitational theories. As in the case of the EinsteinHilbert action one could derive field equations in $\mathrm{f}(\mathrm{R})$ gravity in two different approaches, the so-called metric and Palatini approaches. But in $\mathrm{f}(\mathrm{R})$ actions, unlike the EinsteinHilbert action or its modified version (one with a cosmological constant term), the field equations obtained by the two approaches are not the same in general. In what follows we will be interested only in metric $\mathrm{f}(\mathrm{R})$ theories of gravity in which connection is dependent on metric $g_{\mu \nu}$ with respect to which, the action is varied. In ordinary GR there are not that many exact solutions of the field equations for a given symmetry. Being higher derivative theory it is not unexpected to find more exact solutions in $\mathrm{f}(\mathrm{R})$ gravity and this turns out to be the case for spherically symmetric solutions [3]. Constant curvature solutions in $f(R)$ gravity was studied by Odintsov and collaborators [11]. Also there is a significant review of $f(R)$ and other modified gravities [12] and viable models [13] in literatures. Former Looking for solutions with a different symmetry, we obtained static cylindrically symmetric vacuum solutions of the $\mathrm{f}$ (R) modified Einstein equations [1]. It is shown how one can reduce the set of equations into a single equation which could then be utilized to construct explicit solutions. Now we generalized field equations with the same symmetry For constant curvature solutions, using 
the general form of a cylindrically symmetric solution in Weyl coordinates we find, among possible solutions, a generalized form of zero curvature spacetime as well as one new (nonzero curvature) solutions with one of their parameters chosen so that it is related naively to the cosmological constant. The stability of a non linear system of differential equations is a significance problem. The modern method for study the stability problem is theory of

dynamical systems that first is used by Coley in cosmological models [14]. Folowing Coley we applied this method for our model and obtained a constraint on the initial condition which under it, the system of non linear equations are stable. Our motivation in this paper is that we solved the non linear equations and obtained Tian solution and we prove that our exact solution is applicable to the exterior of a string [5]. All mathematical calculations is done in detail .Therefore this work complete our former paper and although contains some new features of constant curvature solutions in $f(R)$ metric gravity in cylindrical symmetry.Unlike our previous article we do not limit ourselves to a specific gauge, rather we work in a general framework. Finally we present a prediction for the general form of this cylindrically symmetric solution in other modified $f(G), f(R, G)$ models of gravity.

\section{FIELD EQUATIONS IN $f(\mathbf{R})$ GRAVITY}

In this section we give a brief review of the field equations in $f(R)$ gravity. The action for $f(R)$ gravity is given by

$$
S=\int\left(f(R)+\mathfrak{L}_{m}\right) \sqrt{-g} d^{4} x
$$

The field equation resulting from this action in the metric approach, i.e. assuming that the connection is the Levi-Civita connection and the variation is done with respect to the metric $g_{\mu \nu}$, is given by

$$
G_{\mu \nu} \equiv R_{\mu \nu}-\frac{1}{2} R g_{\mu \nu}=\mathrm{T}_{\mu \nu}^{g}+\frac{\mathrm{T}_{\mu \nu}^{m}}{F(R)}
$$

where the gravitational stress-energy tensor is

$$
\mathrm{T}_{\mu \nu}^{g}=\frac{1}{F(R)}\left(\frac{1}{2} g_{\mu \nu}(f(R)-R F(R))+F(R)^{; \alpha \beta}\left(g_{\alpha \mu} g_{\beta \nu}-g_{\mu \nu} g_{\alpha \beta}\right)\right)
$$

with $F(R) \equiv d f(R) / d R$ and $\mathrm{T}_{\mu \nu}^{m}$ the standard matter stress-energy tensor derived from the matter Lagrangian $\mathfrak{L}_{m}$ in the action (1). The vacuum equations of motion, i.e. in the 
absence of matter, are given by,

$$
F(R) R_{\mu \nu}-\frac{1}{2} f(R) g_{\mu \nu} \nabla_{\mu} \nabla_{\nu} F(R)+g_{\mu \nu} \square F(R)=0
$$

Contraction of the above field equations gives the following relation between $f(R)$ and its derivative $F(R)$

$$
F(R) R-2 f(R)+\square F(R)=0
$$

which will be employed later both to simplify the field equations and to find the general form of the $f(R)$ function.

\section{CYLINDRICALLY SYMMETRIC VACUUM SOLUTIONS}

Interested in the static cylindrically symmetric solutions of the vacuum field equations (4), we start with the general form of such a metric in the cylindrical coordinates $(t, r, \varphi, z)$ given by [6];

$$
d s^{2}=-A(r) d t^{2}+d r^{2}+B(r) d \varphi^{2}+C(r) d z^{2}
$$

The corresponding scalar curvature is

$$
R=\sum_{(i \neq j)=1}^{3}\left(\frac{A_{i}^{\prime \prime}}{A_{i}}+\frac{1}{2} \frac{A_{i}^{\prime}}{A_{i}} \cdot \frac{A_{j}^{\prime}}{A_{j}}-\frac{1}{2}\left(\frac{A_{i}^{\prime}}{A_{i}}\right)^{2}\right)
$$

in which ' $\equiv \frac{d}{d r}$ and $A_{i}(r)=A(r), B(r), C(r)$ for $i=1 \ldots 3$ are the metric functions. Using equation (5), the modified Einstein equations become

$$
F R_{\mu \nu}-\nabla_{\mu} \nabla_{\nu} F=\frac{1}{4} g_{\mu \nu}(F R-\square F(R))
$$

As in the spherical case, since the metric only depends on the cylindrical radial coordinate $r$, one can view Eq. (8) as a set of differential equations for functions $F(r), u(r), k(r)$ and $w(r)$. In this case both sides are diagonal and hence we have four equations. Differentiating Eq. (5) with respect to $r$ we have the extra consistency relation for $F(r)$,

$$
R \dot{F}^{\prime}-\dot{R} F+3(\square F)^{\prime}=0
$$

Any solution of Eq. (8) must satisfy this relation in order to be also a solution of the original modified Einstein's equations. From Eq. (8) it is found that

$$
\frac{F R_{\mu \mu}-\nabla_{\mu} \nabla_{\mu} F}{g_{\mu \mu}}=\frac{1}{4}(F R-\square F(R))
$$


In other words the combination $A_{\mu} \equiv \frac{F R_{\mu \mu}-\nabla_{\mu} \nabla_{\mu} F}{g_{\mu \mu}}$ (with fixed indices) is independent of the index $\mu$ and therefore $A_{\mu}=A_{\nu}$ for all $\mu, \nu$. This allows us to write the following independent field equations;

$$
\begin{gathered}
F^{\prime \prime}+\frac{1}{4}\left[\frac{A^{\prime}}{A}\left(\frac{B^{\prime}}{B}+\frac{C^{\prime}}{C}\right)-2\left(\frac{B^{\prime \prime}}{B}+\frac{C^{\prime \prime}}{C}\right)+\left(\left(\frac{B^{\prime}}{B}\right)^{2}+\left(\frac{C^{\prime}}{C}\right)^{2}\right)\right] F=0 \\
F^{\prime}+\frac{1}{2} \frac{B^{\prime}}{B}\left[2\left(\frac{A^{\prime \prime}}{A}-\frac{B^{\prime \prime}}{B}\right)+\left(\left(\frac{B^{\prime}}{B}\right)^{2}-\left(\frac{A^{\prime}}{A}\right)^{2}\right)+\frac{C^{\prime}}{C}\left(\frac{A^{\prime}}{A}-\frac{B^{\prime}}{B}\right)\right] F=0 \\
F^{\prime}+\frac{1}{2} \frac{C^{\prime}}{C}\left[2\left(\frac{A^{\prime \prime}}{A}-\frac{C^{\prime \prime}}{C}\right)+\left(\left(\frac{C^{\prime}}{C}\right)^{2}-\left(\frac{A^{\prime}}{A}\right)^{2}\right)+\frac{B^{\prime}}{B}\left(\frac{A^{\prime}}{A}-\frac{C^{\prime}}{C}\right)\right] F=0
\end{gathered}
$$

corresponding to $A_{t}=A_{r}, A_{t}=A_{\phi}$ and $A_{t}=A_{z}$ respectively. Therefore, any set of functions $F(r), A(r), B(r)$ and $C(r)$ satisfying the above equations would be a solution of the modified Einstein field equations (8). Obviously these equations can not be solved without auxiliary conditions reducing the number of the unknown functions. In the following section we discuss the simple but important case of the solutions with constant curvature.

\section{A. A brief discussion about cylindric constant curvature solution for couple real-} istic $\mathbf{F}(\mathbf{R})$ models

One reason for investigating the constant curvature solution in a typical modified modified $f(R)$ gravity is that, in order that the accelerating expansion in the present universe could be generated, we must consider that $f(R)$ could be a small constant at present universe [20].In this class of models, the universe starts from the inflation driven by the effective cosmological constant at the early stage, where curvature is very large. As curvature becomes smaller, the effective cosmological constant also becomes smaller. After that the radiation/matter dominates. When the density of the radiation and the matter becomes small and the curvature goes to the value $R_{0}$, there appears the small effective cosmological constant. Hence, the current cosmic expansion could start.In the epoch of the inflation, the curvature $R=A$ could be large, we may identify $f(\infty)$ as the cosmological constant for the inflationary epoch and $f\left(R_{0}\right)$ as that at the present accelerating era [20].

\section{B. Constant Curvature solutions in Cylindrical symmetry}

It is known that some of the vacuum constant curvature solutions in $f(R)$ gravity are equivalent to vacuum solutions in Einstein theory with the same symmetry. For example 
it is shown in [4] that in the spherically symmetric case the corresponding $f(R)$ solutions include the Schwarzschild-de-Sitter space for a specific choice of one of the constants of integration. For cylindrical symmetry, in Einstein gravity, static vacuum solutions were found almost immediately after their spherical counter parts by Levi-Civita [6] but those with a cosmological constant have to wait another 60 years to be found by Linet [4] and independently by Tian [5] in a non-Weyl coordinate system. Their solution reduces to that of a cosmic string in the limit $r \rightarrow 0$ [7, 8].

Looking for cylindrically symmetric solutions in $f(R)$ gravity, here we consider the simple but physically important case of static constant curvature spacetimes. To do so, in the field equations (11), (12) and (13) taking $R=$ constant, we arrive at the following set of equations:

$$
\begin{aligned}
& \frac{A^{\prime}}{A}\left(\frac{B^{\prime}}{B}+\frac{C^{\prime}}{C}\right)-2\left(\frac{B^{\prime \prime}}{B}+\frac{C^{\prime \prime}}{C}\right)+\left(\left(\frac{B^{\prime}}{B}\right)^{2}+\left(\frac{C^{\prime}}{C}\right)^{2}\right)=0 \\
& 2\left(\frac{A^{\prime \prime}}{A}-\frac{B^{\prime \prime}}{B}\right)+\left(\left(\frac{B^{\prime}}{B}\right)^{2}-\left(\frac{A^{\prime}}{A}\right)^{2}\right)+\frac{C^{\prime}}{C}\left(\frac{A^{\prime}}{A}-\frac{B^{\prime}}{B}\right)=0 \\
& 2\left(\frac{A^{\prime \prime}}{A}-\frac{C^{\prime \prime}}{C}\right)+\left(\left(\frac{C^{\prime}}{C}\right)^{2}-\left(\frac{A^{\prime}}{A}\right)^{2}\right)+\frac{B^{\prime}}{B}\left(\frac{A^{\prime}}{A}-\frac{C^{\prime}}{C}\right)=0
\end{aligned}
$$

These equations must be solved . One simple assumption is seeking power law solutions for metric functions i.e. $A_{i}(r) \propto r^{m_{i}}$ which leads to 3 simple algebraic equations for $m_{i}$. which as we show in next section this solution and another solutions. In the rest of this section we show that this solution with another set of solutions correspond respectively to a zero and non-zero values of Ricci scalar $R$.

\section{Case (1) : solution with $R=0$}

From the equations (14-16) one could obviously arrange a solution of $A_{i}(r) \propto r^{m_{i}}$ in which the constants $m_{i}$ are given as follows;

$$
\begin{array}{r}
m_{1}=m_{3}=0, m_{2}=2 \\
m_{1}=m_{2}=\frac{4}{3}, m_{3}=-\frac{2}{3}
\end{array}
$$

It can be shown that these are Ricci flat solutions (i.e $R=0$ in (7)) .Indeed in the second case which $m_{1}=m_{2}=\frac{4}{3}, m_{3}=-\frac{2}{3}$ the metric is written as:

$$
d s^{2}=-A_{0} r^{4 / 3} d t^{2}+d r^{2}+B_{0} r^{4 / 3} d \varphi^{2}+C_{0} r^{2 / 3} d z^{2}
$$


by applying the following radial coordinate transformation

$$
r=\frac{4}{3} \rho^{3 / 4}
$$

and choosing the constants $A_{0}=B_{0}=\left(\frac{3}{4}\right)^{4 / 3}, C_{0}=\left(\frac{3}{4}\right)^{2 / 3}$ it transforms into the following historic metric

$$
d s^{2}=-\rho d t^{2}+\rho^{-1}\left[\rho^{1 / 2}\left(d z^{2}+d \rho^{2}\right)+\rho^{2} d \varphi^{2}\right]
$$

it is Levi-Civita's static cylindrically symmetric solution which normally is written in the following general form with the constant $m=\frac{1}{2}$ :

$$
d s^{2}=-\rho^{2 m} d t^{2}+\rho^{-2 m}\left[\rho^{2 m^{2}}\left(d \rho^{2}+d z^{2}\right)+\rho^{2} d \varphi^{2}\right]
$$

That it is obviously flat [10].

\section{Case (2) : solution with $R=$ constant $\neq 0$}

To find solutions of this type we introduce 3 new functions as $A=e^{u}, B=e^{v}, C=e^{w}$. In terms of these functions the equations (11-13) become:

$$
\begin{array}{r}
u^{\prime}\left(v^{\prime}+w^{\prime}\right)-2\left(v^{\prime \prime}+w^{\prime \prime}\right)-\left(v^{\prime 2}+w^{\prime 2}\right)=0 \\
2\left(u^{\prime \prime}-v^{\prime \prime}\right)-\left(v^{\prime 2}-u^{\prime 2}\right)+w^{\prime}\left(u^{\prime}-v^{\prime}\right)=0 \\
2\left(u^{\prime \prime}-w^{\prime \prime}\right)+\left(u^{\prime 2}-w^{\prime 2}\right)+v^{\prime}\left(u^{\prime}-w^{\prime}\right)=0
\end{array}
$$

Solving this equations is complicated. But in a special case $u=v$ equation (24) is satisfied identically and 2 remaining equations $(23,25)$ reduce to:

$$
\begin{aligned}
u^{\prime \prime}-\frac{1}{2} u^{\prime} w^{\prime}+w^{\prime \prime}+\frac{1}{2} w^{2} & =0 \\
u^{\prime \prime}-\frac{1}{2} u^{\prime} w^{\prime}-w^{\prime \prime}-\frac{1}{2} w^{2}+u^{\prime 2} & =0
\end{aligned}
$$

By adding $(26,27)$ we have:

$$
2 u^{\prime \prime}+u^{\prime 2}-u^{\prime} w^{\prime}=0
$$

a straightforward integration gives:

$$
w=2 \ln u^{\prime}+u+c
$$


in which $c$ is a constant . After substituting this solution in (26) we have:

$$
3 u^{\prime \prime}+2 \frac{u^{\prime \prime \prime}}{u^{\prime}}=0
$$

this is a simple 2'nd order ODE w.r.t $u^{\prime}$.Taking $p=u^{\prime}$ and rewriting the derivatives with respect to $u$ we have:

$$
\frac{2}{3} p \frac{d p}{d u}=c-\frac{1}{2} p^{2}
$$

which has the following solution:

$$
p= \pm \sqrt{2 c+A e^{-3 u / 2}}
$$

If we choose only positive sign we can write an explicit form for $u, v, w$ :

$$
\begin{array}{r}
u=v=\frac{2}{3} \ln \left[\frac{A}{2 c} \cos ^{2}\left(\frac{3}{2} \sqrt{\frac{c}{2}} \cdot r\right)\right] \\
w=2 \ln \left[\sqrt{2 c} \cdot \tan \left(\frac{3}{4} \sqrt{2 c} \cdot r\right)\right]+\frac{2}{3} \ln \left[\frac{A}{2 c} \cos ^{2}\left(\frac{3}{4} \sqrt{2 c} \cdot r\right)\right]+c_{2}
\end{array}
$$

Without loss of generality we can set $c_{2}=0$ or absorb it in the $z$ coordinate. If we calculate Ricci scalar (7) with these metric functions we obtain $R=6 c$.So by comparing this with the solutions of the Einstein field equation in the presence of the cosmological constant i.e, $R=4 \Lambda$, we have:

$$
c=\frac{2}{3} \Lambda
$$

Finally we can write the general form of metric (6) as:

$$
\begin{array}{r}
d s^{2}=-\left[\frac{A}{2 c} \cos ^{2}\left(\frac{3}{2} \sqrt{\frac{c}{2}} \cdot r\right)\right]^{2 / 3} d t^{2}+d r^{2}+\left[\frac{A}{2 c} \cos ^{2}\left(\frac{3}{2} \sqrt{\frac{c}{2}} \cdot r\right)\right]^{2 / 3} d \varphi^{2}+ \\
{\left[2 A^{2} c \sin ^{2}(3 / 4 \sqrt{2 c} r) \cos ^{4 / 3}(3 / 4 \sqrt{2 c} r)\right] d z^{2}}
\end{array}
$$

Now we introduce new parameter $\alpha=\left(\frac{A}{2 c}\right)^{2 / 3}$. Our metric solution converts to:

$$
d s^{2}=\alpha \cos ^{4 / 3}\left(\sqrt{\frac{3 \Lambda}{4}} r\right)\left(d \varphi^{2}-d t^{2}\right)+d r^{2}+\left(\frac{4}{3} \Lambda \alpha\right)^{3} \sin ^{2}\left(\sqrt{\frac{3 \Lambda}{4}} r\right) \cos ^{4 / 3}\left(\sqrt{\frac{3 \Lambda}{4}} r\right) d z^{2}
$$

from the general form of Tian metric we have:

$$
\begin{array}{r}
d s^{2}=-[\tan \beta(r+\hat{r})]^{\gamma_{1}}[\sin 2 \beta(r+\hat{r})]^{2 / 3} d t^{2}+[\tan \beta(r+\hat{r})]^{\gamma_{2}}[\sin 2 \beta(r+\hat{r})]^{2 / 3} d z^{2} \\
+[\tan \beta(r+\hat{r})]^{\gamma_{3}}[\sin 2 \beta(r+\hat{r})]^{2 / 3} d \varphi^{2}+d r^{2}
\end{array}
$$


Where $\hat{r}$ is an arbitrary real number, $\beta=\sqrt{\frac{3 \Lambda}{4}}$ and $\gamma_{1}, \gamma_{2} \gamma_{3}$ are real numbers in the interval $\left[-\frac{4}{3}, \frac{4}{3}\right]$, satisfying the algebraic equations

$$
\gamma_{1}+\gamma_{2}+\gamma_{3}=0, \gamma_{1} \gamma_{2}+\gamma_{2} \gamma_{3}+\gamma_{1} \gamma_{3}=-\frac{4}{3}
$$

Easily we observe that our solution is a special sub family of Tian solution with parameters $\gamma_{1}=\gamma_{3}=-\frac{2}{3}, \gamma_{2}=\frac{4}{3}, \hat{r}=0, \alpha=\frac{3 \sqrt{2}}{4 \Lambda}$. Also a change of coordinates (it is better to called them a congruity of non radial coordinates )is needed as:

$$
\varphi \rightarrow\left(\frac{\sqrt{2} \Lambda}{3}\right)^{1 / 2} \varphi, t \rightarrow\left(\frac{\sqrt{2} \Lambda}{3}\right)^{1 / 2} t
$$

Then we prove that in $f(R)$ gravity the constant curvature solution in cylindrically symmetric cases is only one member of the most generalized Tian family in GR, thus we have different kinds of cosmological constant in this scenario and not a fixed one as in GR.

\section{MATHEMATICAL BEHAVIOR OF FIELD EQUATIONS AS AN AU- TONOMOUS DYNAMICAL SYSTEM}

In this section our goal is description stability of system of ODE (23-25) in modern language of dynamical systems [14]. A non linear system of differential equations $X^{\prime}=$ $f_{i}(X), X=\left(X_{1}, X_{2}, \ldots, X_{n}\right), \dot{X}=\frac{d X}{d t}$ is called autonomous . A point $P=\left(X_{1}^{0}, X_{2}^{0}, \ldots ., X_{n}^{0}\right)$ is called a critical point if that be a solution for the system of algebraic equations:

$$
f_{i}(X)=0, i=1,2, \ldots, n
$$

any autonomous system can be spotted near critical point as a linear system. If we construct a matrix $A$ with components $A_{i j}=\left.\left(\frac{\partial f_{i}(X)}{\partial X_{j}}\right)\right|_{P}$, and if the linear perturbation of system from critical point is denoted by $\Psi=\left(\Psi_{1}, \Psi_{2}, \ldots, \Psi_{n}\right)$, then the solution to linearized system $\dot{\Psi}=A \Psi$ determines stability of the original non linear model. If as $t \rightarrow \infty$ all perturbation functions $\Psi_{i} \rightarrow 0$ then we say that the system is stabilized. For our purpose if in system (23-25) we introduce a new set of variables $\xi=u^{\prime}, \eta=v^{\prime}, \theta=w^{\prime}$ and if we denote all of them in form $\xi_{h}, i=1,2,3$ we can write this equations in form :

$$
\frac{d \xi_{i}}{d r}=\frac{1}{2}\left(\xi_{j} \xi_{k}-\xi_{i}^{2}\right), j \neq k, i, j, k=1,2,3
$$


it's related matrix $A$ is:

$$
A_{i j}=\frac{1}{2} \epsilon_{i j k} \xi_{k}-\xi_{i} \delta_{i j}
$$

Where in it $\epsilon_{i j k}$ is a Levi-Civita altering tensor and $\delta_{i j}$ is Kronecher delta function. The critical point of system is located at $P=(\xi, \eta, \theta)=c(1,1,1)$ where in it $c$ is an arbitrary constant. Thus it's value at critical point can be written explicitly by:

$$
A_{i=j}=-c, A_{i \neq j}=\frac{1}{2} c
$$

the eigenvalues of matrix $A$ are:

$$
\lambda_{1}=0, \lambda_{2,3}=-\frac{3}{2}
$$

Thus the general solution for system (42) in linearized aproximation is :

$$
\Psi=\xi_{0}+\left(\eta_{0}+\theta_{0}\right) e^{-\frac{3}{2} r}
$$

For stability we observe that only if $\xi_{0}=0$ the non linear system (42) in linear approximation is stable and consequently the non linear system begins stable.

\section{PHYSICAL RESULTS AND CONCLUSIONS}

In recent decades there has been more interests in Cosmic strings as an Cosmological predictions of String theory. We know that according to the gauge theories with spontaneous symmetry breaking the universe has a number of phase transitions since the big bang. In general, cosmic strings are topological structures which is produced meanwhile in these phase transitions. Later Grfinkle [15] has treated the string as a self-interacting massless minimally coupled scalar field coupled to a U(1) gauge field and has shown that there exists a class of static , cylindrically symmetric solutions to the field equations. Now following the Tian [5] we prove that our metric (37) is applicable to the exterior of a string. This is the main physics which is hidden behind mathematical equations and we elucidate it in the following paragraphes. Consider the exterior metric for a string. One class of exteriors has been discussed by Tian is a solution of equations in a vaccum or by imposing the boost symmetry. The boost symmetry means that in the general Tian metric we must have

$$
\begin{aligned}
\gamma_{1}=\gamma_{2} & = \pm \frac{2}{3} \\
\gamma_{3} & = \pm \frac{4}{3}
\end{aligned}
$$


The result metric is:

$d s^{2}=[a \cos \beta(r+\hat{r})]^{4 / 3}\left(-d t^{2}+d z^{2}\right)+\frac{d^{2} a^{-2 / 3}}{\beta^{2}}[\sin \beta(r+\hat{r})]^{2} \times[\cos \beta(r+\hat{r})]^{-2 / 3} d \varphi^{2}+d r^{2}$

If we apply the complex transformations $t \rightarrow-i \varphi, z \rightarrow i t, \varphi \rightarrow z$, this form accordance with the our metric (37) by choosing parameters $\hat{r}=0, \alpha=\frac{3 \sqrt{2}}{4 \Lambda}, a=\alpha^{3 / 4}, d=\beta a^{1 / 3}$. This proofs that our metric (37) is applicable to the exterior of a string.

Again the motivation for considering material systems with cylindrical symmetry in asymptotically AdS spacetimes comes also from the classical general relativity. it is well known that with $\Lambda=0$, the asymptotes of cylindrically symmetric static spacetimes representing infinite sources is very different from that of spatially bounded static sources. The advantage of the studied spacetimes over the vaccum LC case is that for $\Lambda<0$, far away from the symmetry axis, $r=0$, they approach the anti de Sitter solution (AdS) unlike LC that does not approach the Minkowski spacetime. Lemos [16] found an interesting class of cylindrically symmetric $4 \mathrm{D}$ solutions with $\Lambda<0$ called black strings. These spacetimes describe the fields of charged, rotating strings that generally feature singularities and horizons and are asymptotically anti de Sitter far away from the axis of symmetry. Static plane-symmetric perfect fluids occur also as subcases of the static cylindrically-symmetric solutions, There are several physically interesting classes of solutions admitting an Abelian group $G(2)$ on non-null orbits; because of their importance and the large amount of relevant material, they will be divided into separate parts. The stationary axisymmetric fields have timelike group orbits $T^{2}$. The classes with spacelike group orbits $S^{2}$ Contains time-dependent cylindrically-symmetric fields and their stationary subclasses, colliding plane waves with their typical dependence on the retarded advanced time, and inhomogeneous perfect fluid solutions. Known rigidly rotating axisymmetric perfect fluid solutions with more than two Killing vectors belong to the locally rotationally-symmetric space-times or to the homogeneous space-times or they are cylindrically-symmetric . The stationary cylindrically-symmetric dust solutions are contained in the general stationary axisymmetric dust solution (the Winicour solution) [17]. About generalization of our cylindrically symmetric result for other modified gravities especially for $f(G)$ and $f(G, R)$ one could obtain new families of physically tolerable solution.Former stationary cylindrically symmetric solutions to the five-dimensional Einstein and Einstein - Gauss - Bonnet equations has been investigated and two exact solutions which qualify as cosmic strings, one corresponding to an electrically charged cosmic string, 
the other to an extended superconducting cosmic string surrounding a charged core were found[18]. Thus naturally we expected that in any $f(G)$ model of gravity by imposing the cylindrical symmetry one could be able to recover this rather old family of exact solutions. Note that in $f(G)$-gravity, there are no problems with the Newton law, instabilities and the anti-gravity regime [21]. The analogue of constant curvature in $f(G)$-gravity is $G_{0}$ corresponds to the present value of the Gauss-Bonnet invariant. Technically when $G=G_{0}$ and $G=G_{+\infty}, f(G)$ becomes almost constant and can be regarded as the effective cosmological constant similar to $f(R)$ models. The starting point for doing it is following from which has been done by Nojiri and Odintsov for spatially-flat FRW universe metrics in the context of

modified Gauss-Bonnet $f(G)$ and $f(G, R)$ gravity[19]. As an landscape it seems that for a large number of choices of the function $f(G)$, like that has been discussed in [19, 21] , the field equations has a non-trivial real solution (in which non of metric functions vanishes) and asymptotically converge to a static patch of deSitter universe. We remind it to the another work [22].

\section{ACKNOWLEDGMENT}

D.Momeni thanks S. Nojiri and S. D .Odintsov for useful comments. Finally, the editor of IJMPD, Jorge Pullin and the anonymous referees made excellent observations and suggestions which resulted in substantial improvements of the presentation and the results.

[1] A. Azadi, D.Momeni, M.Nouri Zonoz.Phys. Lett. B., 670 (2008)

[2] H. A. Buchdahl, Mon. Not. Roy. Astr. Soc., 150, 1 (1970)

[3] T. Multamaki and I. Vilja, Phys. Rev. D 76, 064021 (2007)

[4] B. Linet, J. Math. Phys. 27, 1817 (1986)

[5] Q. Tian, Phys. Rev. D 33, 3549 (1986)

[6] T Levi-Civita, Rend. Acc. Lincei 27, 183 (1917)

[7] A. Vilenkin and E. P. S. Shellard, Cosmic strings and other topological defects, Cambridge University Press (1994)

[8] M. Anderson, The mathematical theory of cosmic strings, IOP (2003) 
[9] D. Momeni,A. Azadi.: Astrophysics and Space Science, vol. 317, no. 3.(2008)-pp. 231-234

[10] H. Stephani, D. Kramer, M. A. H. MacCallum, C. Hoenselaers and E. Herlt , Exact Solutions of Einsteins Field Equations, Second edition, CUP (2003)

[11] G Cognola,E Elizalde, S Nojiri, S D Odintsov and S Zerbini, JCAP 0502,2005,010

[12] S Nojiri and S D Odintsov, hep-th:0601213.

[13] S Nojiri and S D Odintsov, hep-th:0807.0685.

[14] A. A. Coley. arxiv: gr-qc /9910074. v1. 21 Oct 1999

[15] D. Grfinkle, Phys. Rev. D 32, 1323 (1985)

[16] Lemos J P S 1995 Class. Quantum Grav. 12 1081; Phys. Lett. B 35346

[17] Winicour, J.(1975). All stationary axisymmetric rotating dust metrics.JMP 16, 1806.

[18] M. Azreg-Ainou and Grard Clment. Class. Quantum Grav. 13 2635-2650, 1996

[19] S. Nojiri, S.D. Odintsov, Int.J.Geom.Meth.Mod.Phys.4:115-146,2007

[20] S. Nojiri, S.D. Odintsov, Phys.Lett.B657:238-245,2007

[21] S. Nojiri and S. D. Odintsov, Phys. Lett. B 631, (2005) 1 arXiv:hep-th/0508049]; S. Nojiri, S. D. Odintsov and O. Gorbunova, arXiv:hep-th/0510183; G. Cognola, E. Elizalde, S. Nojiri, S. D. Odintsov and S. Zerbini, Phys. Rev. D 75, (2007) 086002; I. Brevik and J. Quiroga, arXiv:gr-qc/0610044.

[22] D.Momeni, In preparation. 